LA-UR- 97: 129

\begin{tabular}{l|l} 
Title: & $\begin{array}{l}\text { VIII. THE OBSERVATIONAL } \\
\text { STRATEGY: WHAT ARE THE ISSUES; } \\
\text { WHAT MUST BE DONE? }\end{array}$
\end{tabular}

Author(s): $\quad$ Gregory H. Canavan, DDP

Submitted to:

For discussions with CGCR (Committee on Global Change Research)

Date: January 1997
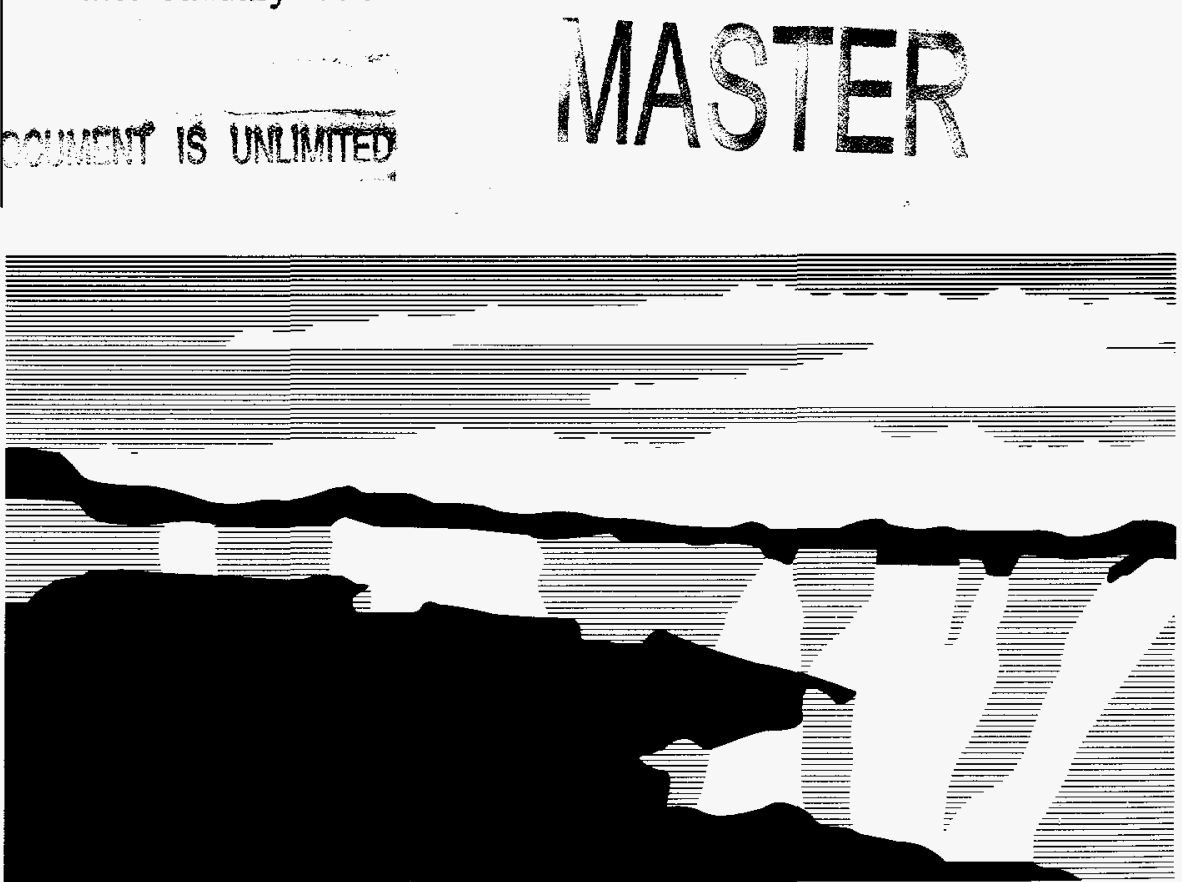

Los Alamos National Laboratory, an affirmative action/equal opportunity employer, is operated by the University of California for the U.S. Department of Energy under contract W-7405-ENG-36. By acceptance of this article, the publisher recognizes that the U.S. Government retains a nonexclusive, royalty-free license to publish or reproduce the published form of this contribution, or to allow others to do so, for U.S. Government purposes. The Los Alamos National Laboratory requests that the publisher identify this article as work performed under the auspices of the U.S. Department of Energy. 


\section{The Observational Strategy: What are the issues; what must be done?}

Remote observation from space has gone through several stages. The 1960 s were a period of technology demonstration. The 1970 s were one of the quantitative measurement of trends and patterns. The $1980 \mathrm{~s}$ made initial quantitative measurements leading to an incipient understanding of the Earth as a system. The 1990s saw the integration of all of these results into the design of an Earth Observing System (EOS) of satellites. The 2000s should see them fly to address many of the issues that remain unresolved today. Through this process, satellite remote sensing matured and became a respected science. It is now in a position to make critical contributions to some of the most important problems in global environmental chance, but doing so will require a careful, selective, and integrated strategy.

Throughout its development, the observational strategy of EOS and its precursor programs has been consistent with that of the Mission to Planet Earth (MTPE) to detect and quantify climate change, document natural climate variability, understand variation and change, determine the causes and impacts of stratospheric ozone depletion, determine the impact of change on ecosystems and mitigate them. Space based observation can contribute significantly to each of these objectives, although its contribution will have to be carefully integrated with aircraft, in situ, international and other contributions and carefully transitioned to long-term operational observations to achieve its maximum potential impact.

The interaction between space and in situ can be in calibration, in interpretation, or in suggesting ways to make important new measurements from space. In atmospheric chemistry it largely involves calibration and global surveys. In ecosystems it involve calibration of EOS and improved sensors. In seasonal to interannual change it involves the testing and calibration of new sensors. In decadal to century change it requires the invention of new sensors. These roles are complementary and reinforcing. Taking full advantage of the synergisms and tradeoffs between space- and ground-based measurements is a potential vehicle for major savings in what is effectively a constant resource program. The only way to satisfy all of the requirements for change measurement and interpretation is to strengthen this interaction and effectively plan and execute

\section{Principles guiding the space-based observational strategy}

As in the other elements of the MTPE, while programmatic goals should be used to prioritize experimental campaigns, the fundamental principle in space-based observations is that science is fundamental basis for decisions on sensors, platforms, programs, and priorities. At each critical juncture the program has been reviewed to assure that the balance of activities reflect evolving scientific priorities. That has required changes in tactics and programs. Priorities have changed as experiments indicated important new directions for research, but the scientific focus of the strategy has not changed. Instead, this flexibility has been maintained through continuing 


\section{DISCLAIMER}

Portions of this document may be illegible in electronic image products. Images are produced from the best available original document. 
redesign and introduction of advancing technology. This flexibility must be maintained if EOS is to be able to identify key scientific questions and how to address them cost effectively. Maintaining such options in a strongly constrained program requires a thorough appreciation of the likely design changes needed over a period of several decades of observations. Maintaining these options affordably will require the imbedding of that integrated observational strategy into the programs for air, in situ, and international observations.

A key element of the space-based observational strategy is assuring that scientific integrity is maintained from process definition to the calibration and validation necessary for maintaining the dynamic continuity of critical long-term measurements through successive generations of sensors. The result is that EOS has state of the art platforms and sensors capable of the best measurements possible of the phenomena accessible to them. Maintaining that strategy through a two-fold reduction in resources has required significant shifts. The most visible is from large to medium and small spacecraft, but there have also been subtle shifts in priority as well. One such shift is a statement of the priority of implementing the first elements of MTPE/EOS (Landsat-7, AM-1, PM1, Chem-1, and TRMM) on schedule over those of the other elements of the program.

It has been necessary to rebalance space observations with in situ observation programs and analysis within a constant total resource base. The reduction in resources for space observations should be compensated for by several innovations. The first is the use of advanced technology to reduce the cost of continuing observations. The second is the use of small satellites and remotely piloted vehicles (RPVs) to reduce the cost of new measurements that are needed such as tropospheric winds and soil moisture. That has called for the integration into EOS of the New Millennium Program (NMP) for spacecraft cost reduction and the ESSP for lower cost advanced observational capabilities such as SARs, lidars, hyper spectral sensors, and tropospheric chemistry sensors. A related goal is to use small satellites to achieve faster turnaround than that possible with large satellites and provide better coupling to defense and commercial technology and applications.

There is a tension in this progression in that the budgets required for planned EOS platforms threaten those for these needed new technologies. Thus, the observation strategy must be fiscally constrained as well. There are three principal vehicles for relieving these strains: domestic convergence, international cooperation, and applications. Converge is exhibited in the incorporation of science requirements into the specifications for DMSP replacement that is to satisfy both DoD and NOAA's requirements for daily operational meteorological coverage through NPEOS. International cooperation is the coordination of the development and flight of spacecraft and sensors. Lack of such coordination is now producing redundancy in sensors in the midst of unnecessary gaps in data sets. Practical benefits of cooperation involve the spin off of EOS sensors and capability to the solution of natural hazards mitigation, water resource management, food and fiber production, and advances in the Earth sciences. These benefits arguably justify the capability 
on their own and could generate private sector contributions of significant magnitude, particularly in the measurement of variables associated with the management of food and fibers.

The overall strategy for space-based observations has been stable for some decades: to take continuing, high quality data sets of all accessible phenomena with improving instruments for a long enough period to make the main contributions from space to each of the four main areas of change studied in MTPE. For that strategy, the initial EOS sensors are more than adequate. However, they need more flexibility, which will require institutional and resource adjustments. It will be necessary to obtain the latter through internal, rebalancing, and international savings, which have not materialized as yet. To attack new measurements will require totally new sensors, which are not available and not programmed.

The first priority of the strategy must be to keep EOS focused and on schedule and to maintain the planning and coordination needed to expedite its coordination. However, even if that is done, the program will have limited flexibility to respond to the initial influx of new and unexpected data from early EOS measurements. Thus, the strategy must retain the capability to respond to new opportunities on the time scale of the individual platforms and sensors. That will require some flexibility in resources, which must be found from within the space observations program. A strategy capable of maintaining the space observations program through its design lifetime of three sets of platforms will have to constantly prioritize and consolidate, transfer essential but expensive measurements to aircraft or ground sensors, and meaningfully integrate planning and execution of measurements by U.S. and international platforms.

\section{a. Atmospheric chemistry}

There are fundamental questions in atmospheric chemistry, e.g., what is the global distribution of ozone in the troposphere and stratosphere, how has it changed recently, and why is the measured depletion in stratospheric ozone so much greater than model predictions? To solve these questions it will be necessary to understand the processes responsible for the transport, production, and loss of trace constituents. Space observations have already made important contributions to these answers by demonstrating the role of active chlorine in the depletion of lower stratospheric ozone and by characterizing the global long-term trends, for which space observation is ideally suited. One would like a deeper understanding, but this correlation is adequate for most controls and agreements.

Ozone illustrates the difficult issues in atmospheric chemistry. The minimum set is a radical, reservoir, and source of each major family $\mathrm{O}_{\mathbf{x}}, \mathrm{NO}_{\mathbf{x}}, \mathrm{CLO}_{\mathrm{x}}, \mathrm{HO}_{\mathrm{x}}$, but the complete chemistry may involve hundreds of species and thousands of reactions, for which only a few dozen rates are known. Moreover, those reactions are spread over gas, liquid, and solid phases in regions that experience significant exchange with the troposphere. A complete solution could involve far greater resources and sensors beyond anything in development. Ultimately, a judgment 
call will be needed between the investment opportunities in chemistry that could lead to useful new understanding but not improve monitoring and the pressing requirements of other measurements.

To give the distribution of ozone in troposphere proper priority, it was necessary to simplify Chem 1-without delaying it-and to focus its tropospheric component on global ozone and its precursors, which should result in a significant advance. UARS doesn't measure the lower stratosphere, which is the region with most uncertainty. CHEM--I will mount four measurements: HIRDLS and MLS will measure tropospheric as well as stratosphere ozone; ODUS, which resembles TOMS, will measure column ozone; and the advanced FTS sensor TES will measure ozone and important precursors in troposphere as well as species in lower stratosphere. This should provide a reasonable understanding and a good global survey. Improved sensors could do even better, but there are alternative ways of providing that understanding.

Ozone illustrates the options for tropospheric measurements. As competitors to satellites, aircraft, balloons, and RPVs offer options for direct sampling and recovery, higher altitude, and longer duration. Even the current ER-2 payload provides in situ measurements of nearly all of the important trace constituents in the lower stratosphere, including the important radical species $\mathrm{OH}$, $\mathrm{HO}_{2}, \mathrm{NO}, \mathrm{NO}_{2}, \mathrm{ClO}$, and $\mathrm{BrO}$. Together with measurements of source and reservoir gases, this payload provides for critical tests of photochemical models. Thus, it appears that aircraft, balloons, and RPVs are preferred for extending trace gas measurements into the troposphere. They are likely to provide the understanding needed. They will also provide the calibration of space-based sensors, which can then be used for global inventories.

Aerosols and clouds have a significant impact on tropospheric chemistry. TOMS and MLS imagery have clearly shown the cooling of the middle and upper stratosphere from the increase in reflectivity caused by aerosols and the reduced absorption of UV light because of reduced ozone. These effects can last for years. Space based sensors can contribute to the understanding of significant issues such as the rate of conversion sulfur dioxide to sulfate aerosols and their residence time. For these studies, EOS AM and PM should be excellent tools. Lidars on free flying small satellites would also be very useful.

The role of fossil fuels, land cover change, and natural ecosystems in controlling trace gases is an important issue. It is significant that the eruption of Mt. Pinatubo was promptly measured, leading to understanding with predictive capability. EOS sensors will further improve the understanding of trends, transport and chemical transformations, aerosols and polar stratospheric clouds.

A strategy for atmospheric chemistry thus involves four parts. The first is the execution and continuity of the EOS platforms - particularly CHEM, which provides the best measurements of atmospheric species and processes possible with current, affordable technology. The second is the monitoring of the stratosphere with evolving sensors for the duration of EOS and beyond. The 
third is a shift in emphasis to the troposphere, accompanied by a shift to in situ measurements for understanding and for calibration of satellites for global surveys. The fourth is the use of the greatly improved calibration of the EOS sensors to reduce the large uncertainty in the radiation budget and chemistry. This combination of developments should provide a major advance, but active measurements will probably be needed. Thus, advanced sensors, including lidars, should be developed in anticipation of that need.

\section{b. Marine and terrestrial ecosystems}

For the prediction and mitigation of the effects of climate change, it is necessary to understand the effects of changing land cover on land-atmosphere exchanges of carbon dioxide, water, and energy; the consequent feedbacks; and how ecosystems respond. These are difficult tasks, but there has been clear progress. It has been shown that it is possible to detect and interpret changes in vegetation. It has also been shown that changes coupled to El Nino can be detected. But there are obvious and important problems. The all-important $\mathrm{CO}_{2}$ inventory has not been established to better than about a factor of two. While the sources and sinks of trace gases are of comparable significance, the $0.9 \% / y r$ growth of methane is not understood--even its cause is not known. Much of the topical rain forest is not monitored. Until these first order issues are addressed, it will not be possible to move with confidence to other important issues, such as the rate of land cover conversion, use intensification, and degradation; how land cover management practices affect water and energy balances; and how EOS sensors can perform repeated global inventories of land-cover and land-use from space.

An progression of capable sensors have been developed to address these issues. AVHRR, Landsat Thematic Mapper (TM) and Spot Panchromatic and Multispectral sensors have shown the utility of space remote sensing of modest spatial, spectral, and temporal resolution. The Lewis Hyper spectral, Clark Panchromatic, and other developmental sensors will explore the extent to which the diagnosis and forecast of land degradation are improved by greater resolution in each of these dimensions. AM-1 with MODIS and MISR, Landsat 7 and later ASTER will provide such extensions. European, Japanese, Canadian, and US SIR-C SARs will significantly extend the quality and extent of the categorization of land cover and biophysical parameters. There is also the option for the use of sensors on commercial satellite constellations to monitor managed ecosystems such as agriculture and forests and to improve temporal resolution.

Overall, there is an embarrassment of riches in platforms and sensors, domestic and international, to address these difficult terrestrial ecosystem questions. Improved resolution and coverage would significantly reduce uncertainties in land cover conversion, intensification, and degradation and the impact of land cover management practices. They can also improve the monitoring of trace gases; however, they cannot do so alone. They will require detailed calibration from in situ measurements. 
There are significant, relevant data sets on ocean parameters. The DMSP SSM/I has several decades of data on integrated liquid water, water vapor, surface wind speed, and sea ice. EOS microwave sensors will extended and improved. What is of need now is information on the biota in oceans. It has been established that it is possible to perform meaningful surveys from space, and it was made a high priority of a dedicated satellite to do so quickly. The delays with Seawifs have impacted those priority measurements. They have also illustrated the problems involved in serial developments of any advanced technology, as are necessary under the EOS resource envelope. Things will inevitably go wrong. It is necessary to plan for that and to keep some options in reserve. In this case, however, it is likely that EOS and international sensors will provide the data needed at higher quality and in duplicate. That illustrates another problem. There is likely to be a glut of ocean color measurements in the next few years, followed by another gap after that. The problem is on of coordination of similar international efforts, which is discussed further below.

Ice sheets, polar and alpine glaciers, and seasonal snow cover the rest of the surface and contain the marginal two percent of the Earth's water that influences much of the vapor budget. Yet current measurements are not sufficiently accurate to determine whether the Antarctic and Greenland ice sheets are growing or shrinking, what their mass balances are, or what the processes are for their growth or thinning. Some information is available from SIR-C, ERS-1 \& -2, Japanese, Canadian SARs, but the launch of the EOS-ALT GLAS will be necessary for measurements of the quality required to determine their mass balances.

The observational strategy: for ecosystems follows directly from the considerations above. Achieving an adequate understanding of carbon and trace gas mass balances will require the calibrated sensors of EOS-AM, Landsat 7, and international SARs and extensive correlation with in situ measurements. Achieving a predictive capability will require visible and infrared sensors, improved to the extent indicated by Lewis and Clark, and SARs improved to the extent indicated by SIR-C/X and TRMM measurements. For maximum impact, these new sensors might be flown quickly on small satellites. Taking advantage of that information will require significant analytical advances. Many of the technical issues in ocean ecosystems should be addressed by Seawifs and EOS, but the institutional issue of minimizing overlap and assuring continuity of international observations remains to be addressed. Adequate monitoring of ice sheets, polar and alpine glaciers, and seasonal snow should be possible with EOS-ALT. The main strategic issue is maintaining the long term continuity of these precise, difficult measurements.

\section{c. Seasonal to interannual timescales}

The overall seasonal to interannual science question can be paraphrased as what mechanisms produce predictability over periods of months to years, where, and with what effects. This implies a more detailed set of needed measurements for different elements.

For the atmosphere, answering these questions require data on atmospheric temperature 
and winds, water vapor, precipitation, sea surface temperature. For an understanding of radiative mechanisms it requires data on cloud and aerosol properties and top of atmosphere radiation. Apart from winds aloft, these are largely the measurements for which EOS-AM and -PM are designed to give global coverage. However, for them to be meaningful, it will be necessary to them to be interpreted and correlated with in situ measurements at the surface.

For the ocean, the essential measurements are sea surface and subsurface temperature, surface winds and precipitation, and sea level, salinity, and ice. These are all difficult measurements, but EOS-AM and -PM and Seawifs are designed to perform all but subsurface measurements. NSCAT has shown the quality of radar surface wind data; Jason-1 should provide continuity of those measurements. TRMM should provide a precipitation measurements that can be calibrated with in situ measurements, although it is necessary to assure the continuity of these measurements to realize their full benefits.

On land, the key measurements are soil moisture, type, and topography; vegetation; temperature, precipitation, runoff, and snow cover. It is also necessary to measure surface radiation coupled with top of atmosphere radiation. AVHRR and Landsat have made indirect measurements of these parameters through indices such as the ratio of bands in NDVI. EOS-AM and -PM should significantly extend the calibration and resolution of those measurements. SSM/I snow and ice measurements should be strengthened by the greatly improved microwave measurements on EOS. ALT and SARs should provide adequate topography. Remote land type and moisture measurements are more primitive. Significantly improving them and the analysis of vegetation will probably require resolutions of the type under investigation in Lewis and Clark. Those extensions are given more importance by the demonstration of the sensitivity of seasonal to interannual phenomenal to antecedent soil moisture.

Radiation budget measurements over land are difficult, but of the order EOS is designed to provide. Perhaps the greatest uncertainty is in measuring the effects of aerosols. SAGE, AVHRR, and TOMS on UARS have demonstrated the ability to measure stratospheric sulfate aerosols. The fuller suite of sensors on EOS should provide better calibrated stratospheric measurements and push the data sets down into the stratosphere, where they can match with in situ measurements. To reduce uncertainties to acceptable levels, it may be necessary to develop active sensors, probably lidars. If so, they might be flown on small satellites.

For change on seasonal to interannual time scales, the basic strategy: appears to be a matter of assuring the continuity of EOS-AM, -PM, and -ALT, and their progressive improvements. For the atmosphere, that would provide most of the measurements required. However, to be meaningful, they would have to interpreted in the light of extensive in situ measurements at the surface to establish the correlations needed for meaningful inventories from space. For the ocean, subsurface temperature and salinity are difficult new measurements. By comparing with in situ 
measurements, it should be possible to develop better correlations. On land, the EOS sensors should improve an already useful capability. Significantly improving measurements of land type and moisture and the analysis of vegetation will probably require resolutions of the type under investigation in Lewis and Clark. If necessary, specialized new sensors might be flown on small spacecraft. The overall strategy is one of assuring continuity of EOS while developing adjunct sensors to better measure winds and subsurface ocean parameters and survey land type, vegetation, and moisture.

\section{d. Decadal to century time scales}

The essential goal in decadal to century change is to assess the extent of climate variability, the causes for them, and to assess their predictable aspects. This task is made difficult by several first order uncertainties. It appears that ENSO drives atmospheric circulation on time scales of decades, but it is not understood how. The rates of change of $\mathrm{CH}_{4}, \mathrm{CO}_{2}$, and $\mathrm{N}_{2} \mathrm{O}$, which are major factors in the radiation budget, currently have no quantitative explanation. And cloud-water vaporization-radiation feedback, which remains the major source of uncertainty in climate change predictions, is not understood in magnitude and possibly in sign.

In this situation perhaps the best that can be done is to continue to better characterize and determine changes in the significant global change forcing factors: solar, carbon dioxide, radiative gases, aerosols, and land cover change by continuous observation. NASA satellites have for some decades been measuring their forcing, feedback, and exchanges of energy, water, carbon. EOS sensors will significantly increase their ability to do so. They should also improve understanding of ocean circulation, the cryosphere, surface fluxes of energy and moisture, and variability. However, the continuation of current efforts is likely to lead to a scientific triage over the next few decades, which will separate problems into the categories: mature, incomplete, and unanswered. A good strategy should be able to determine which category each measurement should fit.

Even for the "mature" problems, it is important to continue to measure many, such as atmospheric irradiance, stratospheric aerosols, total ozone, troposphere water, cloud radiation, and temperature. That will be done with the improved sensors on EOS. It is also appropriate to monitor ocean sea surface temperature, topography, and fluxes, whose measurements will also improve. Land vegetation cover and properties will improve in calibration, although qualitative improvements in discrimination could require somewhat improved sensors. Cryosphere sea ice and continental snow cover will improve with SARs and ALT. Overall, the key requirements is continuity.

The "incomplete" category includes both measurements that are sampled inadequately and those that are not sampled at all. For the atmosphere, they include such measurements as water vapor profiles in upper tropo and lower stratosphere, where EOS sensors loose sensitivity; tropospheric aerosol properties, which may require active sensors; and evaporation, a surface 
process for which no good correlations exist. Ocean surface wind stress measurements have improved with NSCAT, although direct measurements would be useful. Land surface temperature measurements are indirect and of limited accuracy; evaporation is indirect for the reasons described above. Cryosphere ice sheet topography is difficult to measure. Even with EOS-ALT it will be necessary to combine height measurements with inferences of land or ocean motion. Evaporation serves as an example of a measurement that is key to all areas of change but that is very difficult for satellite measurement because it involves both processes above, at, and below the surface and thermodynamic variables for which correlations do not now exist.

The "unanswered" category requires new technologies for missing observations. In the atmosphere that would include global precipitation, which would require dense coverage with active sensors; three dimensional winds, for which affordable space-based lidars or radars do not exist; and cloud microphysics, which would require much greater resolution. Precipitation might be addressed by a simplified, proliferated TRMM, and winds might be partially addressed by current lidars. The other measurements appear to be too difficult to attempt during EOS. However, it is not too early to start research o them. For the ocean, surface salinity is desired, but difficult to measure remotely, and heat flux is needed, but at present can only be inferred from temperature and stress. On land surface soil moisture is needed and could probably be done better with improved sensors. Evaporation and heat flux are difficult for the reasons discussed above. Measurements of cryosphere snow amount or depth and sea ice thickness are limited by the characteristics and coverage of current radars and affordable improvements. In general, the too hard category contains point measurements of gradient phenomena at long distances, which is a different application than those for which the synoptic, imaging EOS sensors are designed. Providing these additional capabilities will require significant additional time and resources.

In summary, the strategy for decadal to century change can be simplified by recognizing that its problems and measurements break down into three rough categories. For the "mature" problems, the main requirement is the continuity of EOS. The "incomplete" measurements require continuity of EOS, evolutionary improvements of its sensors, and better integration with in situ measurements to develop improved correlations for key phenomena such as evaporation. The "unanswered" measurements require more than basic or improved EOS sensors. They generally require active sensors, which have generally proved too demanding for EOS. Evaporation and heat flux require whole new sensors. Perhaps the best possible is a strong correlation from in situ measurements.

\section{e. Human dimensions}

The human dimensions of change involve all of the phenomena observed by EOS, which monitors the changes in land cover, use, and erosion and water availability that permit life; performs high resolution studies of coastal circulation and productivity that support it; and monitor 
the growth of tropospheric pollution that threatens it. The global inventories and correlations that it develops will also improve business and commerce and increase the efficiency of the management of resources. Thus the key element of the observation strategy for human dimensions of change is the continuity of EOS-AM and -PM measurements, although evolutionary improvements in those sensors could provide significant improvements in each of these measurements.

\section{f. Natural hazards mitigation}

The key questions in natural hazards are to understand the reasons for hazards, to use technology to mitigate them, and to coordinate the new capabilities of EOS with other governmental and international efforts. Two promising examples of this process are the dense array of GPS sensors for the measurement of precursors to earthquakes and the development of SAR and SARI processing to detect vertical deformations remotely. In addition, there are the ongoing NASA functions of providing airborne observations of floods with a TRMM sensor, inspections of fires with a infrared sensors related to those on EOS, and locations of fires with AVHRR and Landsat sensors.

NASA has also developed techniques for using the AVHRR sensor to measure or volcanic ash and has developed plans for a small $\mathrm{L}$ band, single polarization LightSAR with a 25 to $250 \mathrm{~km}$ swath for an 8 day revisit time. The higher spatial and spectral resolution of EOS will significantly improve hazard mitigation, particularly in that provision has now been made for the real-time down linking of the information from key sensors. If these measurements prove valuable, their impact could be multiplied by the use faster revisit, small satellites. In each of these activities, current sensors provide a useful capability and EOS will provide a greatly improved capability. Thus, the key to the observational strategy for natural hazards is to maintain the continuity of EOS, particularly the -AM, -PM, ALT, and SAR elements. The benefits to hazard mitigation will be enhanced by strong coupling to in situ measurements. That coupling will also benefit EOS's primary function.

\section{Interplay between space-based and in situ measurements}

The overall interplay between in situ and space observations is conceptually straightforward. Surface based observations are the key to long-term monitoring of chemical changes in atmosphere. They are also the only source of direct understanding of detailed exchange processes at the surface. Airborne measurements are essential for specific processes at various levels in atmosphere. Satellites provide global coverage of chemical such as ozone, processes such as the interaction with ecosystems, and trends such as global change. Thus, space observation has an important role, but space cannot do all of the measurement process by itself, as indicated by the difficulty of measuring reactive nitrogen in troposphere. Within this overall framework, there specifics in the main areas of change, which have been discussed above and are summarized here.

In atmospheric chemistry, the primary interaction between space and in situ observations is 
to calibrate EOS. However, with the shift of emphasis to the troposphere, it will be both possible and necessary for EOS to make much greater use of in situ measurements from aircraft to establish the correlations needed for global maps. Overall, it should be possible to use the much improved EOS sensors to reduce the large uncertainties in the radiation budget and chemistry

Interaction with in situ measurements of ecosystems also starts with calibration. EOS measurements of soil type, moisture, and evaporation are not useful globally unless calibrated locally. Achieving an adequate understanding of carbon and trace gas mass balances will require the full calibration capabilities of the EOS sensors or their improvements. That is also the case with SARs, whose measurements must be calibrated by ground vegetation measurements in tropical rain forests. In ocean measurements, ecosystem interactions will calibrate Seawifs and EOS MODIS sensors. To get the full value of those sensors, it will be necessary to continually improve the correlations used for global surveys. Remote surveys of ice sheets, polar and alpine glaciers, and seasonal snow should be possible with EOS-ALT, but will require extensive ground truth measurements. More comparisons will be required for open ocean ice surveys. Thus, for ecosystems, the main issue is maintaining the long term continuity of the interactions between space and in situ measurements needed to realize the full value of these difficult measurements.

For seasonal to interannual change, the basic interaction is the calibration of EOS-AM, -PM, and -ALT. Atmospheric measurements would require extensive in situ surface measurements. Ocean subsurface temperature and salinity will require new in situ measurements. Land EOS measurements of land type and moisture and the analysis of vegetation will require confirmation as well as calibration. Wind, subsurface ocean, and land type, vegetation, and moisture surveys require coordinated, calibrated in situ measurements rather than new diagnostics.

In decadal to century change, for the "solved" problems, the main requirement is the calibration of EOS. The "can be improved" measurements require calibration of the evolutionary improvements of sensors, and better correlations for key phenomena such as evaporation. The "too hard" problems such as evaporation and heat flux require active sensors. In development they need enough interaction to provide a strong, testable correlation.

For human dimensions, is main interaction is in the calibration and interpretation of the EOS-AM and -PM measurements. For those calibrations to be meaningful, they will have to include global data sets. For natural hazards, the main interaction is in the calibration and interpretation of measurements from EOS-AM, -PM, ALT, and SARs. The benefits to hazard mitigation will be enhanced by strong coupling to in situ measurements, which will also benefit EOS's primary function.

The primary interaction between space and in situ can be in calibration, in interpretation, or in suggesting ways to make important new measurements from space. Atmospheric chemistry largely involves calibration and global surveys. Ecosystems involve calibration of EOS and 
improved sensors. Seasonal to interannual involves the testing of new sensors. Decadal to century requires the invention of new sensors. The only way to satisfy all of these requirements for significant and continuing interaction is to plan and execute space-based and in situ measurements jointly.

A major challenge of EOS with respect to detection and attribution of climate change will be the transition of a research program to an operational monitoring program such that there is a seamless transition of the data records. New and improved measurements are always important, but they must be introduced in such a way that a clear understanding of what they measure, and their biases is known prior to discontinuing the previous instruments or sampling procedures.

3. What are the international issues and how might they be addressed?

The discussion above has, for simplicity, concentrated on U.S. platforms and sensors, but many of those sensors are related to those of international programs. For example, the Japanese Adeos I and II platforms carry visible and infrared, radiation budget, stratospheric and tropospheric chemistry, and active microwave sensors. Moreover, Adeos I caries the U.S. NSCAT and TOMS. The Japanese TRMM will carry visible and IR, radiation budget, active and passive microwave sensors, which will measure precipitation from space for the first time. The follow on HIROS I platform will carry visible and IR imagers, an altimeter, active microwaves, stratospheric and tropospheric chemistry sensors, and possibly a SAR. If so, it would be at a level at or above the complexity of the EOS platforms.

Similarly, Europe is following up its successful ERs-1 and -2 with ENVISAT for the environmental monitoring and atmospheric chemistry and METOP for operational meteorology and climate. It is intended to take over the AM mission from NOAA. This parallel development offers some options for risk reduction. for example, it was intended to perform preliminary studies of aerosol radiative forcing through AVHRR and Seawifs. Given their delay, it has proved possible to substitute POLDER, a French instrument on a Japanese satellite, and GOME on ERS-2. There have been a number of fruitful collaborations such as TOPEX/Poseidon with a U.S. laser on a French satellite for ocean surface height measurements.

The WGCP GEWEX program offers a mechanism for coordinating EOS contributions to fast climate processes in atmospheric clouds and radiation, precipitation, and hydrology, and the CLIVAR/GOALS program gives a similar mechanism for decadal to centennial change contributions. Finally, IGOS provides the outline of how to coordinate and eliminate duplication in all of these international efforts.

Such coordination is essential. At their projected lifetime costs of tens of billions of dollars, the systems are simply becoming too expensive for all of the participants to afford--lead alone for each of the participants to duplicate. The result is that each of the programs is flying sensors too simplified to get needed data and yet still experiencing strains on the technology resources needed 
for future measurements. Some steps are underway, the convergence of the DoD DMSP and NOAA AM platform into NPOES is an essential and important first step. EOS is now in the process of prioritizing future sensor and platform developments.

Similar steps are needed internationally. There are now largely agreed to scientific priorities, and there is sufficient exchange at the technical level to establish a rough consensus on which countries have advantages in various sensor and bus technologies. Formalizing those recognition by assigning leads for various phenomena could provide coverage for all key areas as well as avoiding rampant and obvious duplication that could operate to the detriment of the whole program. EOS has given the U.S. a position of respect in the international arena, which it could use to the benefit of all. However, there are both short and long term impediments. The short term issues are the lack of reliability of space launch agreements over the last few years, which are transitory. The long term issues are the lack of ability to enter into multiyear agreements. Some vehicle for commitments that are binding for at least the life of a spacecraft are necessary if steps towards useful international cooperation are to be realized.

An important by product of such cooperation would be the infusion of the goals of a more resilient, flexible, and faster program for producing better science into world programs, many of which are still in the technology demonstration mode that EOS was in a decade ago. A second by product would be added commitment from each of the participants, which should be reinforcing. The third would be an opportunity to couple international space-based and in situ observational programs in the same manner this discussion is intended to couple them domestically. The fourth would hopefully be enhanced commitments to the technical areas to which each participant subscribed.

\section{Integrated Space-based observational strategy}

The EOS sensors are adequate to the task for which they were designed, and the program is resilient to likely changes in resources. But there will inevitably be surprises, both programmatic and technical. Unfortunately, the planned platforms consume most of resources available, leaving little flexibility for advanced technology, which is developing slowly at its current funding level. Advanced technology is needed for two missions: to lower the cost of continuing observations and to provide improved sensors for the measurement of new phenomena not now known. It has not delivered on either to date. While advanced technology has some initial demonstrations through NMP of its goals of better, cheaper, and faster missions, the ESSP effort to demonstrate similar streamlining of sensor development is in its infancy.

The overall strategy is to take continuing, high quality data sets of all accessible phenomena with improving sensors for a long enough period for space to make the maximum contributions to each of the main areas of change. There are aspects specific to each of the areas of change addressed by MTPE. For atmospheric chemistry, the first is the execution and continuity of the 
EOS, particularly CHEM. The second is the monitoring of the stratosphere with evolving sensors. The third is a shift in emphasis to the troposphere and to in situ measurements for understanding and calibration. The fourth is the use of the improved sensors to reduce the uncertainties in the radiation budget and chemistry. Active sensors will be needed and should be developed.

For ecosystems, an adequate understanding of carbon and trace gas mass balances will require the calibrated EOS sensors, international SARs, and correlation with in situ measurements. Achieving a predictive capability will require the improved visible and infrared sensors, being tested by Lewis and Clark, and SARs, which might be flown quickly on small satellites. Ocean ecosystems should be addressed by Seawifs and EOS. Adequate monitoring of ice sheets, polar and alpine glaciers, and seasonal snow should be possible with EOS-ALT. The main strategic issue is maintaining long-term continuity.

For seasonal to interannual change, the key is assuring the continuity of EOS missions and the progressive improvement of their sensors. For full impact, atmospheric measurements require extensive in situ measurements. For the ocean, subsurface temperature and salinity are difficult new measurements, which require research. EOS should improve an already useful land analysis capability, but significantly improving measurements of land type and moisture and the analysis of vegetation will probably require sensors with new and better resolutions, which might be flown on small spacecraft.

For decadal to century change, the strategy for can be simplified by breaking issues and measurements into three rough categories: solved, for which the main requirement is the continuity of EOS; can be improved, which require evolutionary improvements and better integration with in situ measurements; and too hard, which generally require new and active sensors that have proved demanding in the past. For human dimensions, the key is the continuity of EOS measurements and their evolutionary improvements for transfer and transport. The benefits to hazard mitigation will be enhanced by strong coupling to in situ measurements, which should also benefit EOS's primary function. Improving the interaction between space and in situ measurements involves improving calibration, interpretation, and ways to make important new measurements from space. Closer international cooperation would produce a more resilient, flexible, and faster program for producing better science with added commitment from each participant, which should couple international space-based and in situ observational programs more strongly.

The first priority of the strategy must be to keep EOS focused and maintain schedule. An essential part of that is the planning and coordination needed to expedite its coordination. Even if that is done, the program will have limited flexibility to respond to the initial influx of new and unexpected data from early EOS measurements. The strategy must retain the capability to respond to new opportunities on the time scale of the individual platforms and sensors. That will require some flexibility in resources, which must be found from within the program through prioritization 
and consolidation. It may also require the transfer of some essential measurements to aircraft or ground sensors. It will surely require meaningful integration of the planning and execution of measurements by U.S. and international platforms.

\section{DISCLAIMER}

This report was prepared as an account of work sponsored by an agency of the United States Government. Neither the United States Government nor any agency thereof, nor any of their employees, makes any warranty, express or implied, or assumes any legal liability or responsibility for the accuracy, completeness, or usefulness of any information, apparatus, product, or process disclosed, or represents that its use would not infringe privately owned rights. Reference herein to any specific commercial product, process, or service by trade name, trademark, manufacturer, or otherwise does not necessarily constitute or imply its endorsement, recommendation, or favoring by the United States Government or any agency thereof. The views and opinions of authors expressed herein do not necessarily state or reflect those of the United States Government or any agency thereof. 